Ann. Abeilles, \%, (3), 2I7-23I.

\title{
RECHERCHES SUR LES VARIATIONS DU COMPORTEMENT DE BUTINAGE CHEZ DES COLONIES D'ABEILLES DE RACES DIFFÉRENTES
}

\author{
Z. WARAKOMSKA et J. LOUVEAUX \\ Stalion de Recherches sur l'Abeille et les Insectes sociaux, \\ Bures-sur-Yvette (Seine-et-Oise)
}

SOMMAIRE

Les auteurs ont étudié la récolte du pollen de to colonies d'abeilles appartenant à deux races différentes en utilisant la méthode classique de la trappe à pollen. Sur le plan quantitatif, on note une tendance des abeilles hybrides (Apis mellifica mellifica $\times$ Apis mellifica ligustica) à récolter davantage de pollen que les abeilles de race noire pure $(A . m$. mellifica $)$. Qualitativement, on constate des différences qui sont statistiquement significatives.

\section{INTRODUCTION}

On sait que la récolte du pollen par les abeilles peut être étudiée de façon satisfaisante en utilisant la trappe à pollen, instrument déjà décrit (LoUvEAUx, I958) et dont l'emploi a permis de dégager quelques lois essentielles du butinage. (TodD et Bishor, I940 ; ECKert, I942 ; Synge, I947 ; Maurizio et Kollmann, I949; HIRSCHFEL,DER, I95I ; LOUVEAUX, I958; WARAKOMSKa, I962).

Avec le présent travail, nous avons voulu reprendre l'étude des différences de comportement de butinage susceptibles d'exister entre des colonies de races différentes. On sait déjà que de telles différences existent (Louveaux, I958) mais les problèmes qu'elles posent sont encore incomplètement résolus. C'est pourquoi nous nous sommes proposés de réaliser une étude comparative portant sur le butinage aux divers moments de la journée et en utilisant à la fois des colonies de race noire et des colonies hybrides. 


\section{MATÉRIEL, ET TECHNIQUES}

On a utilisé pour les expériences qui vont être décrites deux lots de ruches d'origine distincte. Un premier lot fut constitué par 5 colonies d'abeilles noires (Apis mellifica mellifica) originaires de la région parisienne et ne présentant aucun signe d'hybridation avec une race autre que la race noire. Un second lot comprenait 5 colonies d'abeilles présentant des caractères morphologiques indiquant sans équivoque possible une hybridation du type $A . m$. mellifica $\times A$. m. ligustica. Ces colonies provenaient de notre station d'apiculture expérimentale du Vaucluse et avaient été amenées ì Bures-sur-Yvette au début de décembre 1962.

Au début du printemps 1963 , les dix colonies expérimentales furent installées sur un même emplacement à la Station de Bures-sur-Yvette. On prit soin d'alterner les colonies des deux groupes de façon à supprimer tout effet de position susceptible de favoriser un lot par rapport à l'autre.

Des trappes à pollen en position supérieure du type déjà décrit par LAvik et FRESNAYE (1963) ont été mises en place au début d'avril sur les io ruches expérimentales. I.es récoltes de pollen obtenues n'ont cependant fait l'objet d'une étude précise que pendant sept journées (les 19, 22, $2.3,24$ avril $, 6,7,8$ mai i 963 ) choisies en raison de conditions météorologiques suffisamment favorables. Pour chacune de ces journées, nous avons procédé à trois prélèvements, le premier à $10 \mathrm{~h}$, le second à $\mathrm{I} 3 \mathrm{~h}$ et le troisième à $\mathrm{I} 6 \mathrm{~h}$; chacun de ces prélèvements correspondait à une période de récolte d'une durée de 20 minutes, c'est-i-dire, en gros, Io h-10 h $20 ; 13 \mathrm{~h}-13 \mathrm{~h} 20 ; 16 \mathrm{~h}-16 \mathrm{~h} 20$. Étant clonné le décalage d'environ une heure entre l'heure légale et l'heure solaire vraie de Buressur-Yvette, on peut considérer que les prélèvements ont été faits à $9 \mathrm{~h}, \mathrm{I} 2 \mathrm{~h}$ et $15 \mathrm{~h}$, heure solaire vraie.

Pour chacune des 7 journées d'observation précédemment citées, nous disposons donc de trois échantillons de la récolte de chacune des dix ruches, soit 30 échantillons par jour et 2 Io échantillons en tout. (En fait, la récolte ayant été nulle à différentes reprises, le nombre total des échantillons utilisables n'a été que de I 70 ). Chaque échantillon a été séché à l'étuve à $35^{\circ} \mathrm{C}$ jusqu'à l'obten. tion d'un poids constant, ce qui exigeait environ 2 à 3 jours. On a procédé ensuite au triage des pelotes par espèces botaniques et les lots de pollen homogène ont été pesés séparément. Cette méthode était donc sensiblement celle qui a été précédemment décrite par l'un d'entre nous (LovveAUx, 1958). Toutefois, chaque échantillon ne représentant qu'un poids minime de pollen, on a pu procéder au triage de la totalité des pelotes. Toutes les identifications ont été faites au microscope selon les techniques habituelles.

Parallèlement, nous avons procédé à la pesée des ruches au début et à la fin de l'expérience, ainsi qu'à la mesure des surfaces de couvain selon la méthode de Fresnaye (ig62).

Tous les résultats obtenus ont été soumis au calcul statistique. Nous avons utilisé le test $t$ (différence entre deux moyennes) et pris $v=8$, le nombre des colonies étant de 5 dans chacun des deux lots. Tous les pourcentages ont subi une transformation angulaire en degrés, selon la formule $\varphi=$ arc sinus $\sqrt{\frac{\varphi}{100}}$.

\section{PRINCIPAUX RÉSULTATS}

Les résultats obtenus au cours de ce travail sont de plusieurs ordres. Les uns concernent les quantités de pollen récoltées et le développement des colonies : les autres concernent le rythme journalier du travail de récolte ou la nature des plantes utilisées par les abeilles.

\section{I. - Résultats quantitatifs}

L'un des buts de ce travail était de comparer le rythme de développenent des colonies de race noire et des colonies hybrides. Comme éléments de comparaison, nous disposons du poids des ruches au début et à la fin de l'expérience, des mesures de surfaces du couvain et $\mathrm{du}$ poids des récoltes de pollen. Nous avons regroupé toutes ces données dans le tableau I dont nous allons faire l'analyse. 
a) Poids des ruches.

Au I7 avril I963, toutes les ruches ont un poids assez voisin de $27 \mathrm{~kg}$. Les différences individuelles sont relativement peu importantes et le poids total des deux lots est sensiblement le même : I38,500 $\mathrm{kg}$ contre $138 \mathrm{~kg}$. Au cours de la période considérée pour notre expérience, les variations de poids sont très faibles par rappcrt à la normale; les conditions météorologiques, particulièrement défavorables jusqu'en avril, ont beaucoup retardé le développement des colonies et par voie de conséquence les augmentations de poids. Celles-ci sont un peu plus fortes chez les colonies hybrides que chez les noires mais la différence n'est pas statistique ment significative $(t=0,740)$.

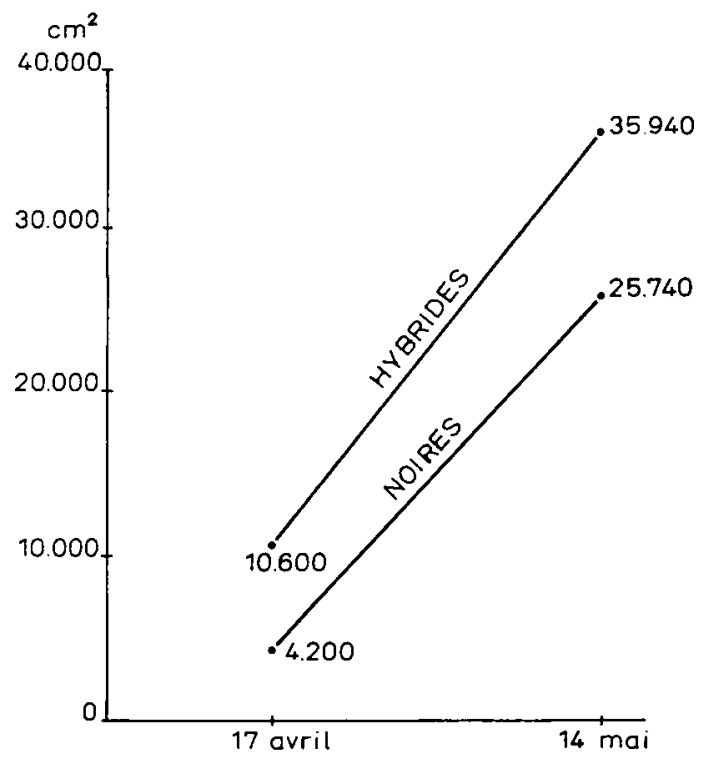

FIG. I. - Erolution des surfaces de couvain ches les colonies de race noire et cher les colonies hybrides.

b) Surfaces de couvain.

Au I7 avril, on note que les colonies sont, dans l'ensemble, petı développées. Ceci est, comme nous venons de le voir, la conséquence d'un hiver long et rigoureux dont l'action se fait sentir très tard en saison. Les colonies noires sont moins développées que les hybrides. Entre le I7 avril et le I4 mai, le développement est important pour toutes les colonies mais les hybrides présentent un grain de surface du couvain plus fort que celui des colonies noires. La figure I montre la différence de pente des courbes d'accroissement pour chacun des lots. Dans l'ensemble, l'avance présentée par les colonies hybrides se maintient au lieu de s'estomper. Toutefois, les différences individuelles sont importantes.

c) Récolte du pollen.

Les observations sur la récolte du pollen ne portent que sur une période très restreinte : 6 heures en tout entre le I 9 avril et le 8 mai. On ne peut donc pas relier 
directement le poids de cette récolte au développement des colonies, d'autant plus que certaines anomalies apparaissent nettement ; la ruche 5 I9 par exemple, qui se distingue par une très forte récolte n'est pas celle qui présente le plus fort accroissement des surfaces de couvain; il en est de même pour la ruche 6I. Il s'agit-là de différences individuelles dont l'origine est incertaine. Sur l'ensemble des colonies, on note cependant une tendance assez nette chez les hybrides à récolter davantage de pollen : $192 \mathrm{~g}$ contre $\mathrm{I} 32 \mathrm{~g}$ pour les noires. On note également une différence dans le rythme des récoltes ; cette différence peut être mise en évidence par le calcul ci-après.

Soient $\mathrm{P}$ et $\mathrm{P}^{\prime}$ les poids totaux de la técolte du pollen des colonies de race noire et des colonies hybrides. Soient $p_{1}, p_{2}$, $p_{3}$, etc. et $p_{1}^{\prime}, p_{2}^{\prime}, p_{3}^{\prime}$, etc. les poids correspondants des récoltes journalières. On calcule :

$$
\frac{p \mathrm{I} \times 100}{\mathrm{P}}, \quad \frac{\left(p \mathrm{r}+p^{2}\right) \text { I00 }}{\mathrm{P}}, \quad \frac{\left(p \mathrm{I}+p^{2}+p_{3}\right) \text { I00 }}{\mathrm{P}}, \text { etc. }
$$

et

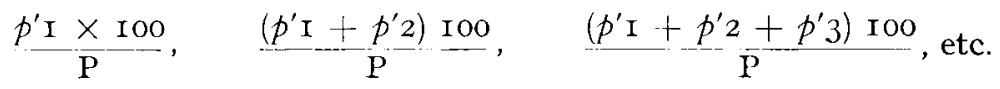

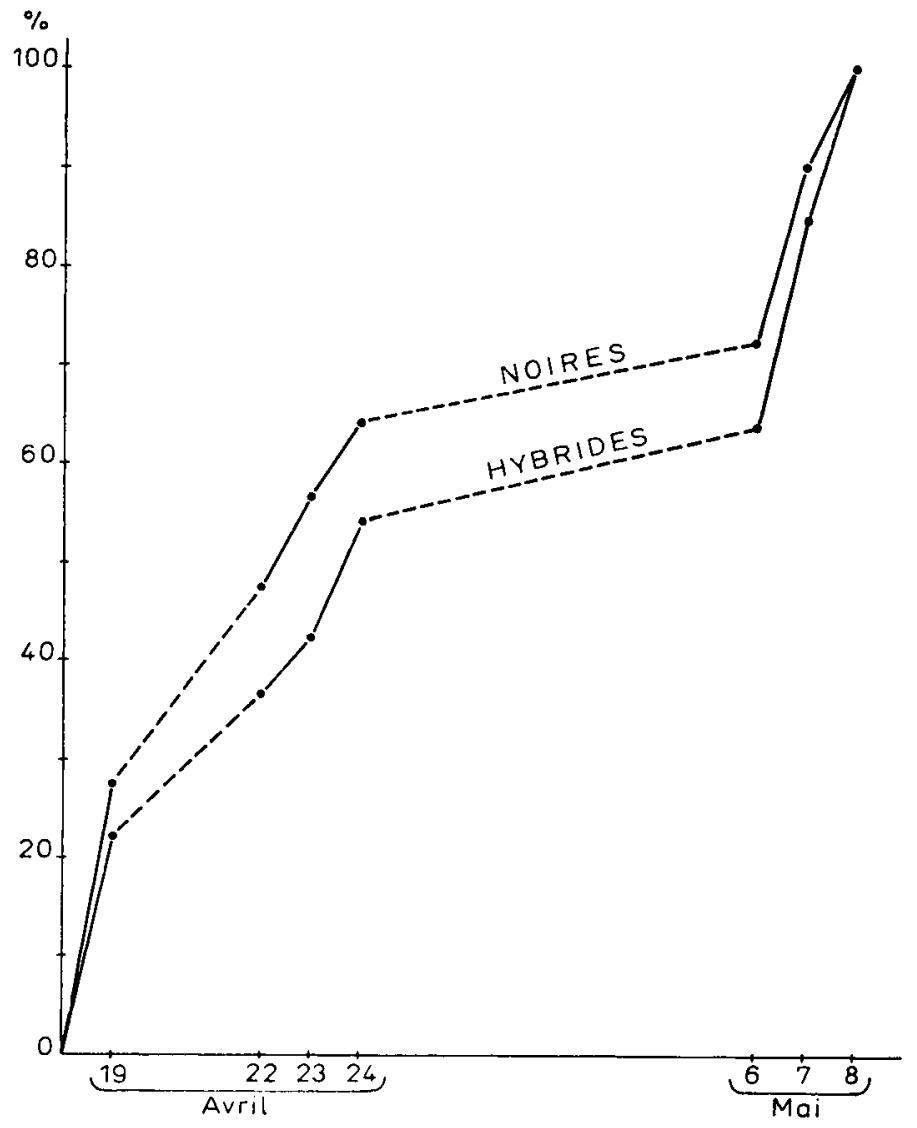

FIG. 2. - Différences du rythme de la récolte du pollen entre les colonies de race noire et les colonies hybrides 


\begin{tabular}{|c|c|c|c|c|c|c|c|c|c|c|c|c|}
\hline 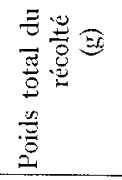 & $\frac{\text { 总 }}{\frac{1}{4}}$ & $\begin{array}{l}\text { â } \\
\text { on } \\
\text { on } \\
\rightarrow\end{array}$ & 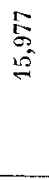 & 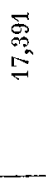 & 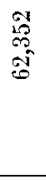 & 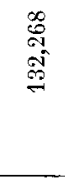 & $\underset{0}{\stackrel{8}{0}}$ & $\begin{array}{l}\overline{\delta_{5}} \\
\text { oi } \\
\text { si }\end{array}$ & $\begin{array}{l}\text { s. } \\
\text { is } \\
\text { is }\end{array}$ & $\underset{+}{\stackrel{9}{8}}$ & $\begin{array}{l}\sqrt{D} \\
\infty \\
\text { S } \\
\text { N }\end{array}$ & $\begin{array}{l}5 \\
\text { o } \\
\text { oi } \\
2\end{array}$ \\
\hline 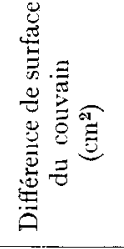 & $\begin{array}{l}9 \\
+ \\
+\end{array}$ & $\begin{array}{l}\stackrel{9}{10} \\
\infty \\
\infty \\
+\end{array}$ & $\begin{array}{l}\stackrel{\rho}{\sigma} \\
\infty \\
+\end{array}$ & $\begin{array}{l}\underset{1}{2} \\
\infty \\
+ \\
+\end{array}$ & $\begin{array}{l}8 \\
8 \\
+ \\
+\end{array}$ & $\begin{array}{l}\text { 早 } \\
\text { is } \\
+\end{array}$ & $\begin{array}{l}\stackrel{9}{2} \\
+ \\
+\end{array}$ & $\begin{array}{l}\stackrel{\infty}{-} \\
+ \\
+\end{array}$ & $\begin{array}{l}8 \\
8 \\
\sigma \\
\sigma \\
+\end{array}$ & $\begin{array}{l}\underset{\mathbb{E}}{\stackrel{8}{0}} \\
+\end{array}$ & $\begin{array}{l}8 \\
0 \\
+ \\
+\end{array}$ & $\begin{array}{l}\stackrel{P}{1} \\
\text { is } \\
+ \\
+\end{array}$ \\
\hline 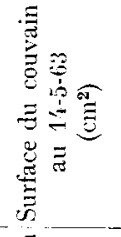 & 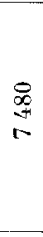 & $\begin{array}{l}8 \\
8 \\
0 \\
++1\end{array}$ & $\underset{8}{8}$ & $\begin{array}{l}\text { @ิ } \\
\text { s } \\
\infty\end{array}$ & $\underset{20}{\stackrel{8}{:}}$ & $\begin{array}{l}\text { \% } \\
\text { in } \\
\text { an }\end{array}$ & $\underset{\infty}{\stackrel{\infty}{\infty}}$ & 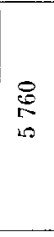 & $\begin{array}{l}9 \\
0 \\
0 \\
+1\end{array}$ & $\underset{\infty}{\stackrel{\Xi}{0}}$ & 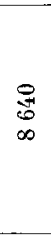 & 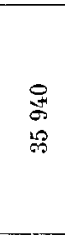 \\
\hline 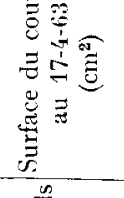 & 冚 & $\underset{\infty}{\mathscr{\infty}}$ & $\therefore$ & $\stackrel{8}{1}$ & $\stackrel{8}{\stackrel{8}{\sigma}}$ & 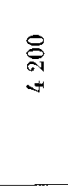 & $\underset{\sim}{\stackrel{\rho}{*}}$ & $\begin{array}{l}\mathbb{D} \\
\text { ज } \\
\text { जv }\end{array}$ & $\begin{array}{l}\text { Dి } \\
\text { oN } \\
\text { ov }\end{array}$ & 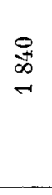 & 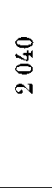 & $\begin{array}{l}8 \\
\$ 0 \\
0\end{array}$ \\
\hline 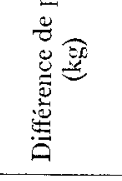 & + & $\theta$ & 0 & 0 & 0 & + & $\begin{array}{l}\stackrel{5}{+} \\
+\end{array}$ & $\begin{array}{l}o 1 \\
+\end{array}$ & 0 & $\begin{array}{l}\stackrel{5}{7} \\
+\end{array}$ & $\begin{array}{l}o r \\
+\end{array}$ & + \\
\hline 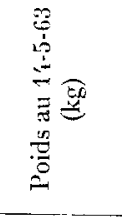 & 8 & 今2 & ลू० & 29 & $\stackrel{g}{\circ}$ & $\underset{\sim}{\stackrel{\Xi}{\sim}}$ & $\stackrel{5}{\circ}$ & $\therefore$ & $\stackrel{\infty}{\sim}$ & $\stackrel{8}{\circ}$ & $\overrightarrow{\sigma 0}$ & $\begin{array}{l}10 \\
\text { 盟 }\end{array}$ \\
\hline 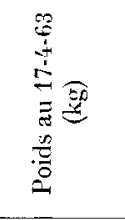 & 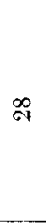 & $\begin{array}{l}20 \\
\mathrm{a}\end{array}$ & 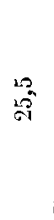 & $\stackrel{2}{\circ}$ & 8 & $\stackrel{\infty}{\stackrel{\infty}{\sim}}$ & : & 22 & 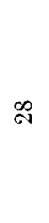 & 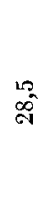 & 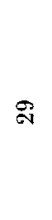 & 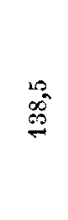 \\
\hline$\frac{\mathscr{0}}{3}$ & $\begin{array}{c}\vdots \\
\vdots \\
\vdots \\
\vdots \\
\vdots \\
2\end{array}$ & $\begin{array}{c}\vdots \\
\vdots \\
\vdots \\
\vdots \\
\frac{9}{20} \\
z\end{array}$ & $\begin{array}{l}\dot{2} \\
z \\
z\end{array}$ & $\begin{array}{l}\stackrel{5}{8} \\
\dot{z}\end{array}$ & $\begin{array}{c}\vdots \\
\vdots \\
\vdots \\
\vdots \\
\text { क्ष } \\
\text { z }\end{array}$ & 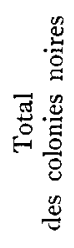 & $\begin{array}{c}\vdots \\
\vdots \\
\vdots \\
\vdots \\
8 \\
8\end{array}$ & $\begin{array}{r}\vdots \\
\vdots \\
\vdots \\
\vdots \\
\text { is } \\
\text { घ }\end{array}$ & $\begin{array}{l}\vdots \\
\vdots \\
\vdots \\
\vdots \\
5 \\
\text { एे }\end{array}$ & $\begin{array}{c}\vdots \\
\vdots \\
\vdots \\
\vdots \\
8 \\
0\end{array}$ & $\begin{array}{c}\vdots \\
\vdots \\
\vdots \\
\vdots \\
\vdots \\
-1 \\
-1\end{array}$ & 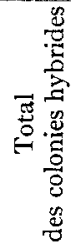 \\
\hline
\end{tabular}


On porte en ordonnées les pourcentages ainsi obtenus et en abscisses les dates de récolte (fig. 2). On peut alors tracer deux courbes qui mettent en évidence le rythme de la récolte. On constate que les ruches de race noire ont récolté au début de la période considérée un plus fort pourcentage du total que les ruches hybrides. Ceci signifie que les hybrides récoltent le pollen en quantités de plus en plus fortes au fur et à mesure de l'écoulement du temps alors que les noires ont tendance à conserver un rythme plus régulier; autrement dit, l'accélération de la récolte est moins forte chez les noires que chez les hybrides.

En résumé, l'examen des données du tableau I montre qu'en moyenne les hybrides ont montré une tendance à un développement plus rapide que les noires. Il ne s'agit que d'une tendance mais on la retrouve aussi bien dans l'évolution du poids que des surfaces de couvain ou des récoltes de pollen. Si aucun élément ne bénéficie d'une confirmation nette par le calcul statistique on peut cependant constater que l'ensemble est cohérent et traduit une certaine aptitude des hybrides à un développement plus rapide au début de la saison apicole.

\section{2. - Variations de la récolte aux divers moments de la journée}

On sait que la récolte du pollen ne se fait pas de façon constante au cours de la journée. Elle est généralement plus forte le matin que le soir ; ce phénomène est en rapport avec l'offre qui dépend elle-même du rythme d'éclosion des fleurs. Il était donc intéressant de savoir si toutes les colonies suivent de la même façon le rythme de récolte imposé par les végétaux ou bien si certaines colonies plus douées sont capables de s'en affranchir dans une certaine mesure. Nous avons donc calculé pour chaque ruche en expérience le pourcentage du pollen récolté aux trois moments de la journée : le matin, midi et soir. Les résultats sont consignés dans le tableau 2

TABLEAU 2

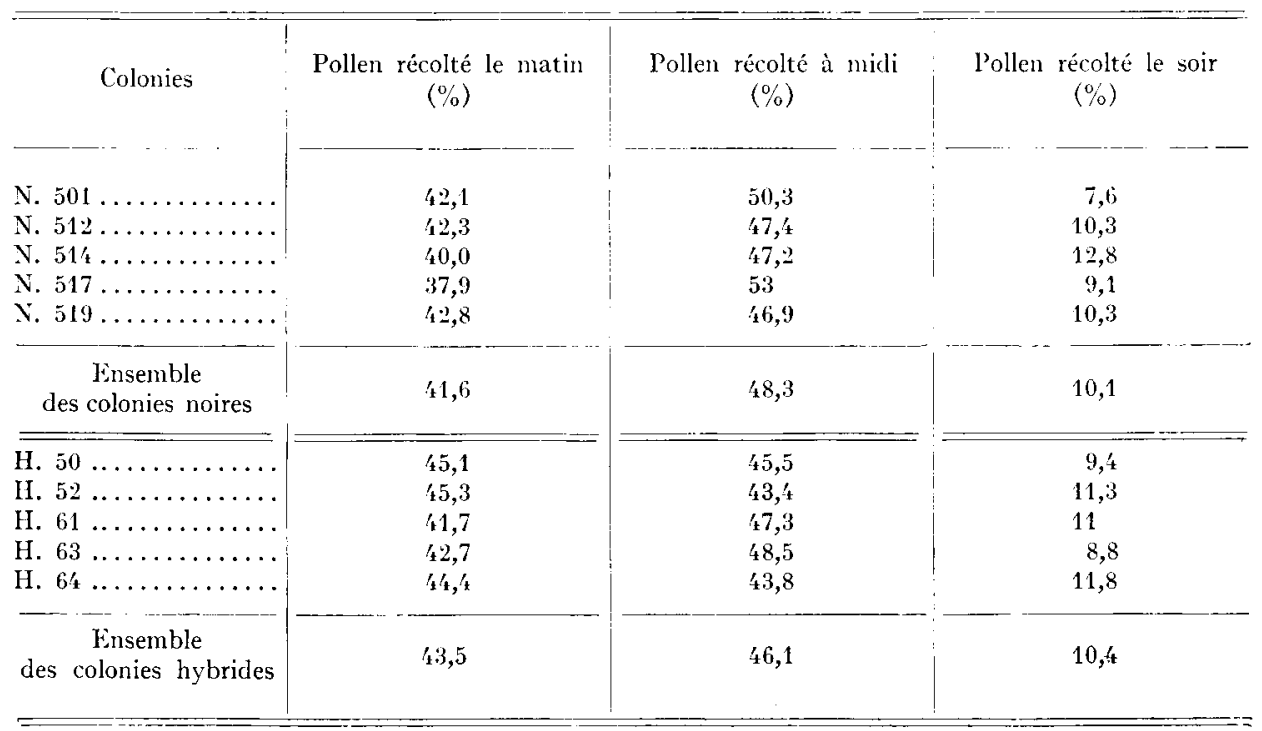


On constate que la dispersion de ces résultats est faible. La légère différence en faveur des hybrides pour la période matinale $(43,5 \mathrm{p}$. Ioo contre $4 \mathrm{I}, 6 \mathrm{p}$. Ioo) est trop faible pour qu'on puisse lui trouver une signification réelle

On doit donc admettre que 1'activité des colonies se répartit entre les différents moments de la journée selon un même schéma. Cette constatation suggère une importance primordiale du facteur végétal. Les abeilles suivent, grosso modo, le rythme de l'offre du pollen par les plantes. Nous verrons plus loin qu'il y a lieu de compléter ces conclusions.

\section{3. - Résultats qualitatifs}

L'analyse des lots de pollen récoltés ayant été faite de façon très détaillée, nous disposons d'une masse de résultats assez importante pour qu'il soit nécessaire d'opérer une subdivision.

Il nous a paru intéressant de rechercher si l'on pouvait observer des différences de comportement notables entre les colonies de race noire et les colonies hybrides

a) La flore utilisée.

TABLEAU 3

\begin{tabular}{|c|c|c|c|c|c|c|c|c|c|c|c|c|}
\hline & 501 & 512 & 514 & 517 & 519 & $\begin{array}{l}\text { Total } \\
\text { noires }\end{array}$ & 50 & 52 & 61 & 63 & 64 & $\begin{array}{c}\text { Total } \\
\text { hybride }\end{array}$ \\
\hline Arbres fruitiers ......... & 17 & 16 & 17 & 17 & 17 & 84 & 17 & 17 & 17 & 17 & 17 & 85 \\
\hline Salix sp $\ldots . . . \ldots \ldots \ldots$ & 17 & 17 & 17 & 15 & 17 & 83 & 15 & 17 & 17 & 16 & 17 & 82 \\
\hline Taraxacum officinale...... & 2 & 5 & 4 & 3 & 6 & 20 & 8 & 4 & 5 & 6 & 5 & 28 \\
\hline Acer pseudo-plalanus...... & 5 & 4 & 5 & 5 & 5 & 24 & 5 & 6 & 6 & 4 & 6 & 27 \\
\hline Enlymion nutans...... & 3 & 3 & 4 & 3 & 6 & 19 & 3 & 6 & 6 & 2 & 6 & 23 \\
\hline Quercus sp. $\ldots \ldots \ldots \ldots$ & 2 & 5 & 3 & 2 & 3 & 15 & 2 & 4 & 4 & 3 & 3 & 16 \\
\hline Fagus silvatica $\ldots . . . \ldots$. & 1 & 1 & 2 & 1 & 6 & 11 & 1 & — & 2 & - & 1 & 4 \\
\hline Anemone nemorosa ........ & 2 & 2 & - & - & - & ' & 2 & - & 1 & - & 2 & 5 \\
\hline Sinapis anensis $\ldots \ldots \ldots$ & - & 一 & 2 & - & 1 & 3 & 3 & 3 & 2 & 1 & 2 & 11 \\
\hline Tussilago farfara ........ & - & 1 & 1 & 1 & - & 3 & - & 1 & 2 & - & - & 3 \\
\hline Aesculus hippocastunum .. & - & $\ldots$ & - & - & 2 & 2 & 1 & - & 2 & 1 & - & 4 \\
\hline Hyacinthuss sp. .......... & - & 1 & - & - & -.. & 1 & 1 & - & - & - & - & 1 \\
\hline Monocotyledone X......... & 1 & 一 & - & - & - & 1 & - & 1 & - & - & - & 1 \\
\hline Acer negindo............ & - & - & - & - & - & - & - & 1 & - & $\ldots$ & - & 1 \\
\hline Ulmus campestris......... & - & - & - & 2 & - & 2 & - & 2 & 1 & - & 1 & 4 \\
\hline Acer sp................ & - & - & - & - & - & - & - & - & 1 & - & - & 1 \\
\hline Betula alba............ & - & 一 & - & - & - & - & - & - & 1 & - & - & 1 \\
\hline Ficaria rununculoides .... & -- & - & - & - & 1 & 1 & - & - & 2 & - & - & 2 \\
\hline Populus nigra $\ldots \ldots \ldots \ldots$ & - & 一 & - & — & $\cdots$ & - & - & $\ldots$ & 1 & - & - & 1 \\
\hline Viscum album $\ldots \ldots \ldots \ldots$ & - & - & $\ldots$ & - & 1 & 1 & - & - & 1 & - & - & 1 \\
\hline Cardamine pratensis ...... & - & - & - & - & - & - & - & 1 & -- & 2 & - & 3 \\
\hline Fragaria iesca............ & 1 & - & - & - & - & 1 & - & 1 & 't & - & - & 5 \\
\hline Lonicera $\mathrm{sp}, \ldots \ldots \ldots \ldots \ldots$ & 1 & 一 & - & - & - & 1 & 1 & - & - & - & - & 1 \\
\hline Syringa vulgaris ........ & 1 & - & - & - & - & 1 & t & 2 & - & - & - & 6 \\
\hline Acer platanoides ......... & - & - & - & - & - & 一 & 一 & - & - & 1 & - & 1 \\
\hline Brassica napus .......... & 一 & - & - & - & - & - & 1 & - & - & - & 1 & 2 \\
\hline Larix europaeus ......... & - & - & 1 & -- & - & 1 & - & 一 & - & - & -- & - \\
\hline (N) Total .............. & 53 & 55 & 56 & 49 & 65 & 278 & $6{ }^{\prime}$ & 66 & 75 & 53 & 61 & 319 \\
\hline Nombre des espèces visitées & 12 & 10 & 10 & 9 & 11 & 20 & 14 & $1^{\prime} \mathrm{k}$ & 18 & 10 & 11 & 26 \\
\hline Rapport $\mathrm{P} / \mathrm{N} . . . \ldots \ldots \ldots$ & 0,45 & 0,22 & 0,28 & 0,35 & 0,96 & 0,17 & 0,49 & 0,50 & 0,79 & 0,77 & 0,46 & 0,60 \\
\hline
\end{tabular}


à l'égard de la flore du lieu où se déroulait l'expérience. On savait déjà (LOUvEAux, I958) qu'il existe de telles différences de comportement liées au patrimoine héréditaire et à l'état physiologique des abeilles. Il n'était pas sans intérêt de vérifier les résultats antérieurs en utilisant les données expérimentales fournies par le présent travail.

Rappelons tout d'abord que nous disposons de 17 analyses de récoltes par colonie, chaque analyse correspondant à l'une des périodes précédemment décrites. Dans le tableau 3 nous avons porté, en regard de la liste complète des plantes dont

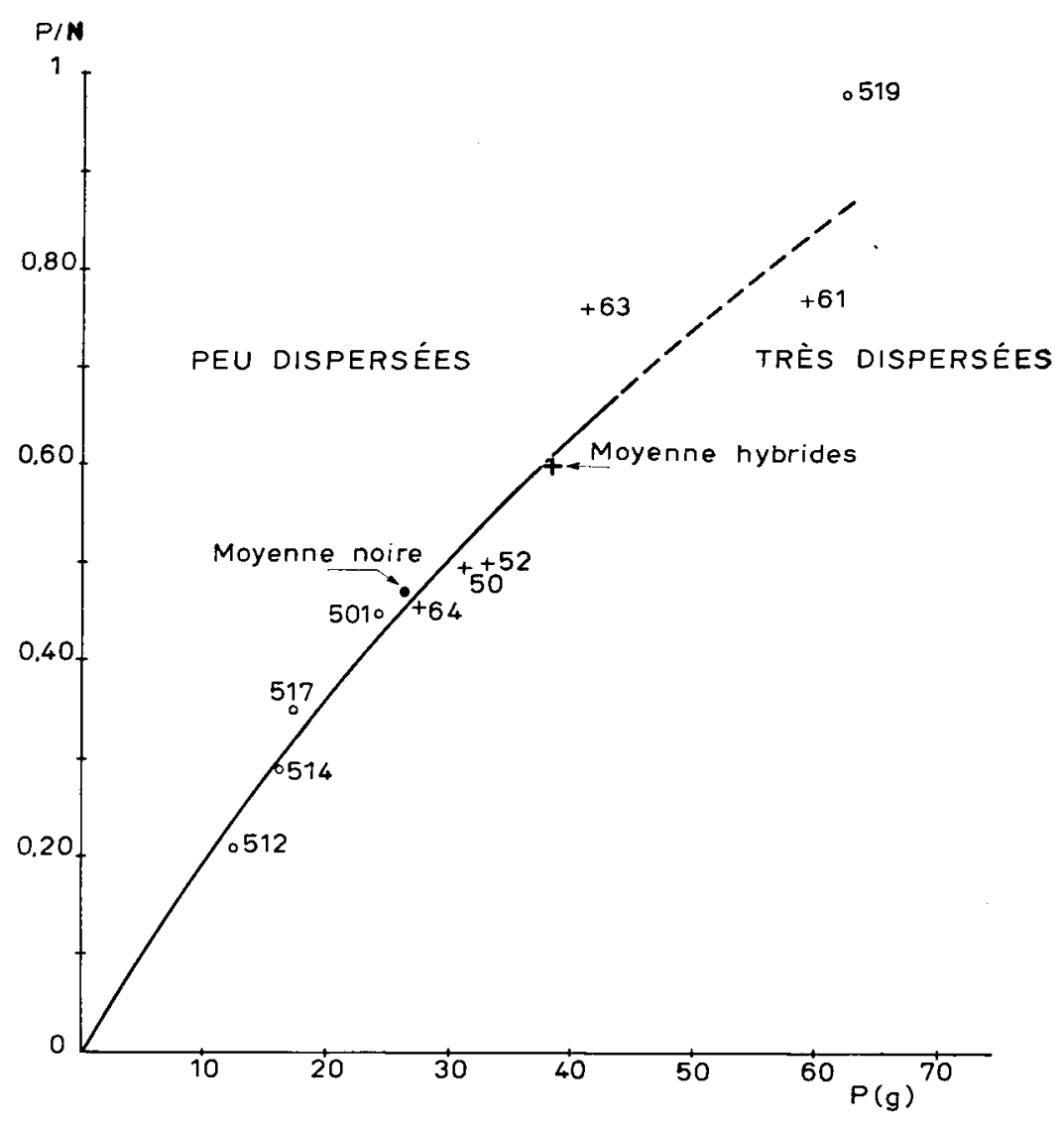

FIG. 3. - Caractìre de plus on moins grante dispersion des récoltes chez les différentes rucle's en expérience

le pollen a été identifié dans les récoltes, le nombre des analyses dans lesquelles le pollen en question figura. On obtient de cette façon, pour chaque colonie, un chiffre qui reflète exactement l'état de fractionnement de la récolte analỹsée. On note par ailleurs le nombre total des espèces utilisées au moins une fois.

L'examen du tableau 3 montre que le nombre total des espèces végétales utilisées est plus fort chez les hybrides que chez les noires ( 26 contre 20 ). Ce résultat est significatif $(t=2,242)$. Par ailleurs, la dispersion est plus grande : 3I9 fractions 
contre 278. Mais ces derniers chiffres ne sont pas vraiment comparables. En effet, il faut tenir compte du fait que plus un échantillon est volumineux plus on a de chances d'y trouver un nombre élevé d'espèces. La relation n'est pas linéaire puisque le nombre des espèces possibles n'est pas infini ; on peut toutefois considérer qu'il existe, en gros, un rapport direct de proportionnalité dans la zone qui nous intéresse. Nous avons donc calculé le rapport $\frac{\mathrm{P}}{\mathrm{N}}$ où $\mathrm{P}$ est le poids de la récolte et $\mathrm{N}$ le nombre des fractions obtenues. Ce rapport représente le poids moyen des fractions de la récolte Sur la figure 3, nous avons porté en abscisses le rapport $\frac{\mathrm{P}}{\mathrm{N}}$ et en ordonnées le poids de la récolte. Nous obtenons une série de points sensiblement alignés qui nous permettent de tracer une ligne de démarcation de part et d'autre de laquelle se situent les ruches à activité relativement dispersée et les ruches à activité relativement concentrée. Le tracé de cette ligne, est bien entendu, empirique.

Dans l'ensemble, toutes les colonies s'alignent sensiblement le long de la déniarcation. Seules font exception les colonies 63 et $6 \mathrm{I}$; la première se révèle comme très concentrée, la seconde comme très dispersée. Le comportement de la ruche 5 I 9 est incertain en raison de sa position par rapport à la courbe dans une zone où le tracé n'est pas sûr. Une différence entre les deux lots de ruches n'apparaît pas nettement. On ne trouve que des différences individuelles qui intéressent telle ou telle colonie, indépendamment du groupe. On peut tout juste soupçonner une tendance plus forte à la dispersion chez les colonies hybrides.

Notons encore que si la flore utilisée par les abeilles en I963 à Bures-sur-Yvette n'est pas rigoureusement la même qu'en I953, I954 ou I955 (LoUvFAux, I958), les différences sont minimes et ne portent que sur des plantes d'intérêt très secondaire.

b) Différences de comportement vis-à-vis des principales sources de pollen.

Nous venons de voir qu'il ne semble pas exister de différence systématique entre les colonies de race noire et les colonies hybrides en ce qui concerne la dispersion sur un nombre d'espèces végétales plus ou moins élevé. Les plantes utilisées sont sensiblement les mêmes mais on peut se demander si leà abeilles les exploitent toujours de la même façon; autrement dit, trouverons-nous toujours la même proportion des différents pollens dans les récoltes des différentes ruches ou groupes de ruches.

Il se trouve que, dans la période étudiée, deux groupes de plantes mellifères jouent un rôle prépondérant : les Arbres fruitiers et les Saules. Sous le terme "Arbres fruitiers " nous regroupons les Rosacées des genres Prunus et Pirns c'est-à-dire essentiellement les Cerisiers, Pruniers, Poiriers et Pommiers. Les Saules sont représentés surtout par l'espèce Salix capraea mais également par tous les autres Saules, d'ailleurs peu nombreux, susceptibles d'exister autour de la Station.

Mettant à profit la composition très simplifiée des récoltes de pollen pendant la période des expériences, nous avons regroupé sous une même rubrique (" divers ") toutes les plantes secondaires et nous avons dressé le tableau 4 . Dans ce tableau, les pourcentages sont calculés sur le poids total de la récolte de pollen. On note inmmédiatement une tendance très nette des ruches hybrides à utiliser davantage les Arbres fruitiers que les Saules et une tendance inverse chez les ruches de race 
noire. La différence est importante puisqu'elle atteint environ ro p. Ioo sur la moyenne générale; cependant la dispersion des valeurs individuelles est grande. C'est pourquoi nous avons soumis nos chiffres au calcul statistique. Ce calcul montre que les différences sont significatives; pour le Saule on trouve $t=2,40$ (pour $v=8$ et après correction angulaire) et pour les Arbres fruitiers $t=1,98$. La différence entre les deux valeurs de $t$ selon qu'on considère le cas des Saules ou celui des Arbres fruitiers s'explique par le fait que les "divers" sont plus importants chez les hybrides que chez les noires. Les colonies hybrides ont donc une tendance très nette à récolter moins de pollen de Saule que les noires, davantage de pollen d'arbres fruitiers et davantage de pollens divers.

TABIEAU 4

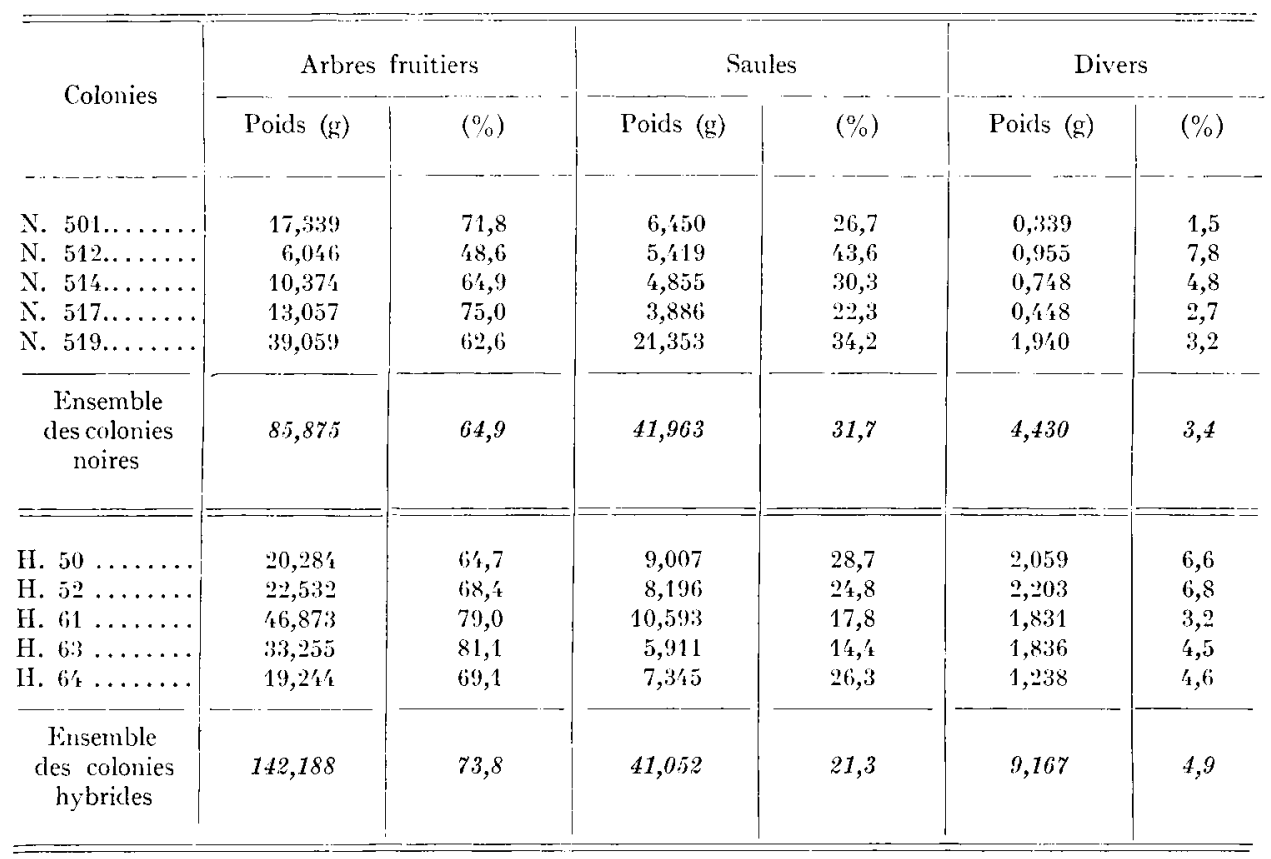

Disposant de données analytiques complètes permettant une étude de la composition des récoltes en fonction du moment de la journée, nous les avons mises à profit pour rechercher si la préfétence des colonies hybrides pour le pollen des arbres fruitiers et la préférence des colonies noires pour le pollen de Saule se manifeste de la même façon le matin, à midi et le soir. Les pourcentages qui figurent au tableau 5 sont calculés de la façon suivante pour chaque colonie :

$$
\text { Poids du pollen } x \text { dans les récoltes du matin (midi, soir) } \times \text { Ioo. }
$$

On constate que pour toutes les colonies, le pollen des arbres fruitiers est récolté en proportion croissante du matin vers le soir. Le pollen de Saule est récolté surtout le matin ; le rythme est inverse de celui des arbres fruitiers. Ces résultats sont statis - 


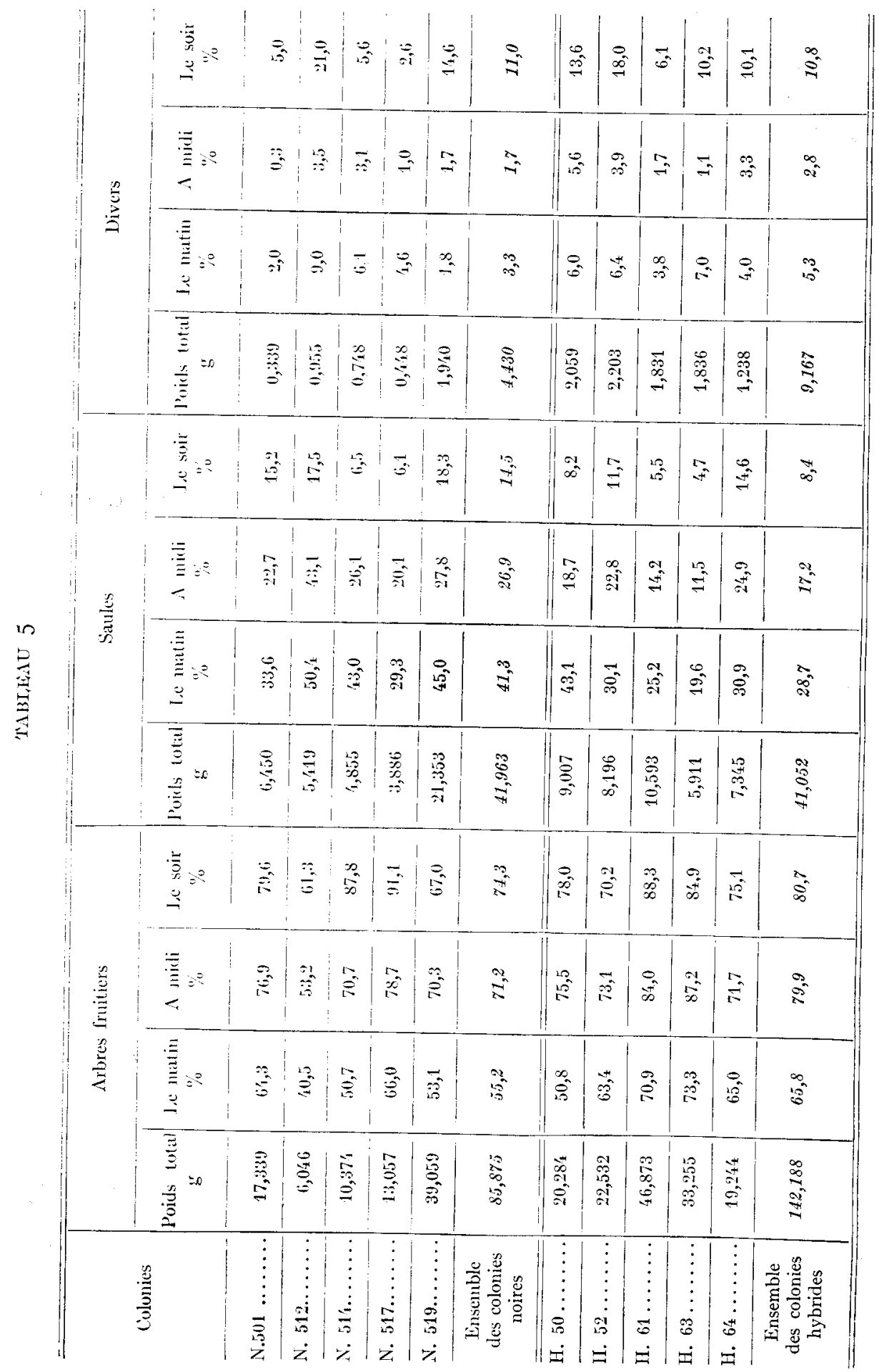


¿iquement significatifs. Pour les divers, on ne peut tirer aucune_règle générale, ce qui est compréhensible puisqu'il s'agit de plantes très variées ayant chacune un rythme d'offre du pollen qui lui est particulier.

Dans l'ensemble, on peut dire que l'examen des données du tableau 5 confirme les constatations qui découlent de l'étude du tableau +. Les différences de comportement apparaissent encore plus accentuées lorsqu'on découpe la récolte en tranches horaires. Si les colonies hybricles récoltent proportionnellement plus de pollen d'arbres fruitiers que les colonies noires, cette différence n'est rraiment très significative que le matin; elle a tendance à s'estomper au cours de la journée. De même, les colonies noires ne récoltent proportionnellement plus de pollen de Saule que dans la première noitié de la journée. Il en découle que l'augmentation de récolte sur les arbres fruitiers croît plus rite au cours de la journée pour les abeilles noires que pour les hybrides. Réciproquement, la diminution sur les saules est plus sensible chez les noires.

Il est intéressant de noter que les différences de comportement sont plus accusées au moment où la plante présente sa production la plus forte; la polarisation des colonies sur une plante déterminée trouve alors son expression maximum. Au fur et à mesure de l'épuisement des ressources en pollen, les différences ont tendance à s'atténuer.
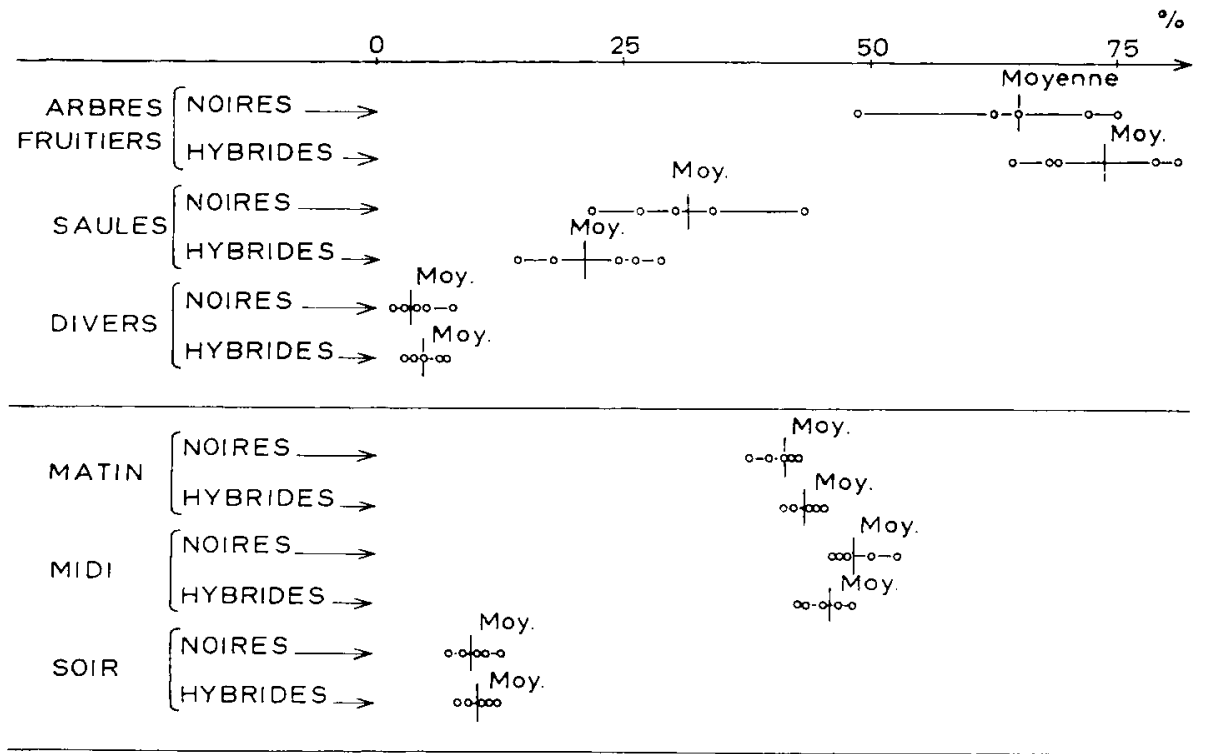

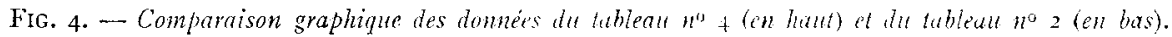

Les pourcentages obtenus par les calculs précédents ont pour inconvénient de ne pas rendre compte des quantités de pollen effectivement récoltées. Le pollen d'arbres fruitiers se rencontre dans la proportion de 75 à 80 p. Ioo dans les récoltes du soir mais il faut savoir que celles-ci sont très réduites ell poids. C'est pourquoi dans le tableau 6 nous avons opéré une nouvelle série de calculs; cette fois nous effectuons :

$$
\frac{\text { Poids du pollen } x \text { dans les récoltes du matin (midi, soir) } x \text { roo }}{\text { Poids total du pollen } x \text { dans les récoltes }}
$$




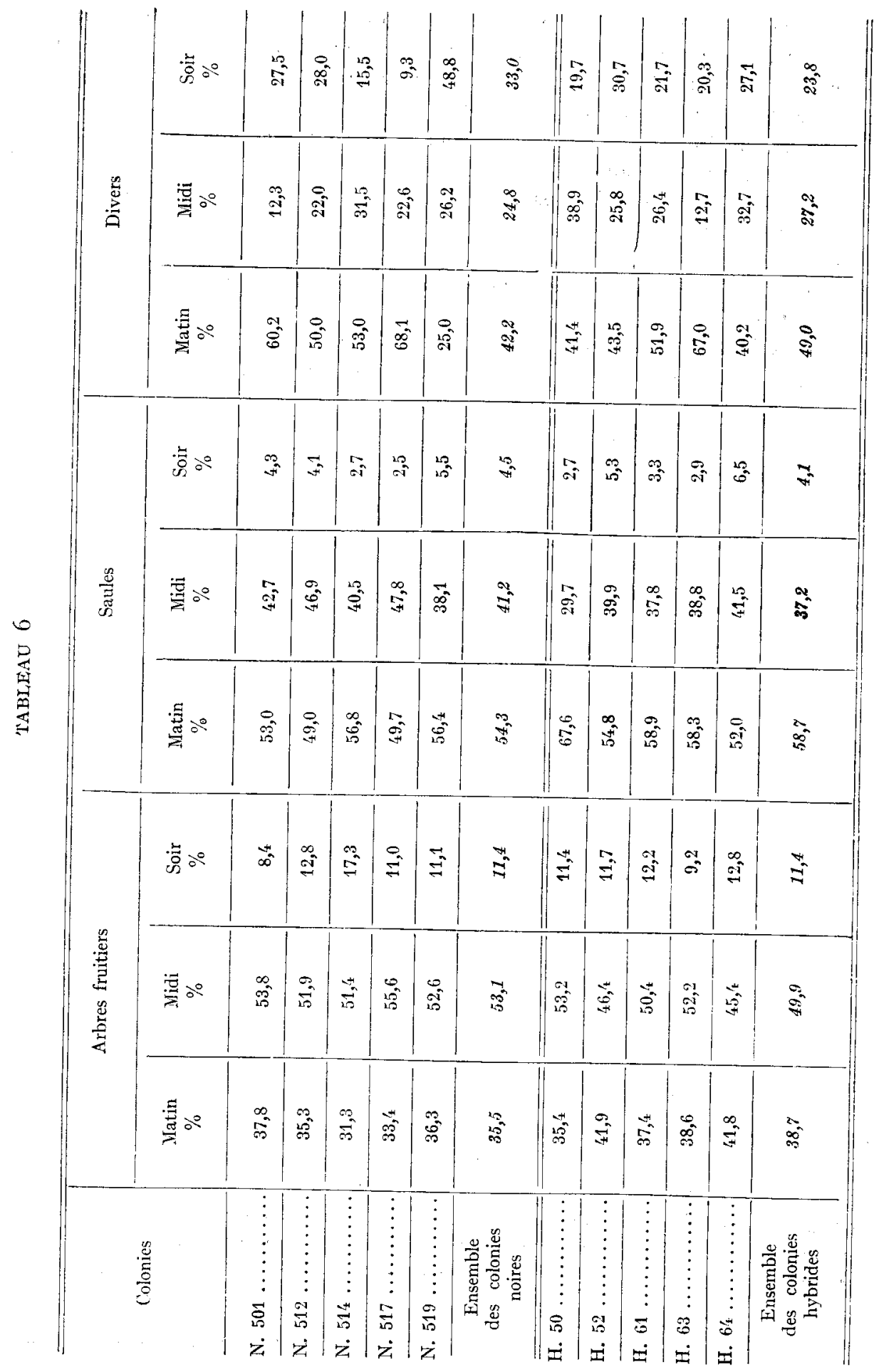


Il ressort du tableau 5 que les chiffres obtenus présentent beaucoup plus d'homogénéité que ceux des tableaux 4 ou .5. Ils sont à peu près aussi homogènes que ceux du tableau 2. Nous avons d'ailleurs tenté de mettre en éviđence le contraste des données par le graphique de la figure 4.

On constate donc que pour un pollen donné le rythme de la récolte par les abeilles varie peu d'une ruche à l'autre ou d'un groupe de ruches à l'autre. Le rôle du végétal comme régulateur de l'activité des abeilles apparaît clairement. Le pollen est récolté en fonction de l'offre; celle-ci est plus matinale chez les Saules que chez les Arbres fruitiers qui dispensent leur pollen aux heures les plus chaudes de la journée. Les phénomènes de polarisation des abeilles sur telle ou telle source font que celle-ci est exploitée plus activement qu'une autre mais le rythme de l'exploitation au cours de la journée reste conditionné avant tout par le végétal.

Bien que les données du tableau 6 soient relativement homogènes on notera que les ruches hybrides ont tendance à travailler plus tôt le matin que les noires. Il ne s'agit que d'une tendance et non pas d'un phénomène vraiment significatif. Nous trouvons confirmation de la tendance qui s'exprime par les données du tableat1 2.

\section{DISCUSSION}

I a comparaison des récoltes de pollen, l'étude des variations de poids et l'obserration du développement du nid à couvain chez des colonies de race noire et chez des colonies hybrides, nous a amenés à faire un certain nombre de constatations. L es colonies hybrides présentent une tendance générale à la précocité qui se manifeste par un développement plus important et plus rapide et par la récolte d'une quantité supérieure de pollen. Parallèlement, on constate une tendance à utiliser un plus grand nombre de sources de pollen et surtout à préférer le pollen des arbres fruitiers à celui des Saules. Cette dernière tendance avait déjà été observée (LoUVEAUX, I958) dans d'autres conditions ; on avait noté alors que l'utilisation préférentielle du pollen de Saule caractérise certaines colonies. On avait constaté aussi que le choix des pollens à haute teneur en azote est le fait des colonies les plus actives et les plus développées, ce qui se trouve à nouveau confirmé, le pollen d'arbres fruitiers étant très riche en azote.

L'importance du facteur végétal dans le rythme journalier de la récolte du pollen a été mise clairement en évidence par l'analyse fractionnée des récoltes. On a pu confirmer le fait déjà établi par MaUrizio et Kolimann (I949) que le pollen des arbres fruitiers est offert pendant une grande partie de la journée ; par contre, nos résultats divergent en ce qui concerne les Saules.

Dans l'ensemble et compte tenu des résultats antérieurs, on est amené à penser que les différences de comportement entre abeilles noires et abeilles hybrides sont liées à des différences d'activité; les abeilles hybrides se sont montrées plus actives que les noires et plus aptes à utiliser au maximum les meilleures sources de pollen. 


\title{
REMERCIEMENTS
}

Nous remercions M. Philipp: Vergikon qui a bien voulu se charger des calculs statistiques font il est fait mention dans le présent travail.

\section{SUMMARY}

\author{
INVENTIGATIONS OF TIIE VARIATIONS IN POLLEX GATIERING MEHAVIOLR \\ IN COLONILS OF IMFLERENT STRAINS
}

The experiment was carried out with two lots of hives:

5 colonies of black bees of pure strain $(A, m$. mellifica) and 5 colonies of hybrid bees (A. M. melli. fro $X$ A.m. ligustica). On the average, the hybrids showed a tendency towards more rapid development than the black bees, both in the development of the weight of the hives and in the brood-cell surfaces or the anrounts of pollen gathered. 'The qualitative results are statistically significant. 'The' total number of plant species used is greater with hybrids than with black bees, with a tendencr: to dispersion with the former. The hybrid hives make use of fruit trees arther than willows. whilst the hives of the black strain have the opposite tendency.

\section{RÉFÉRENCES BIBLIOGRAPHIQUES}

ECKERT J., 1942. Pollen requirement by a colony of llomeybees. J. econ. Entom., 35 (3), 309-311.

FrFavise, J., 1962. In appareil pour le alcul rapide des surfaces de couvain dans les ruches. Am. Abeille, $5(2), 145^{-153}$.

IHRschfeliner II., I95I. Quantitative Untersuchungen zum Polleneintragen der Bienenvölker. Z. Bienenforsch. 1, 54, 67-7\%.

Lavie l'. et Fresnaye J., 1963. Étude expérimentale de la trappe à pollen en position supúrieure. Ann. 1beille, 6 (4), $277-301$.

Lorveltx J., I95\%. Recherches sur la récolle du pollen par les Abeilles (Apis mellifica L.). Thése, P'aris, I-206.

Malrizio A. et Kof,mane II., r949. Pollenanalytische Entersuchumgen an Honig und Poilenhöschen. C. Iscolwhhtungen an Pollenhöschen. Schacis. Bienenstg (Beihefte), 2 (18), 442-455.

SYYGE A. 1)., I947. Pollen collection by Honcybees. J. animal Ecol.. 16 (2), I22-139.

ToLn et lisisop, i944. Ie rôle du pollen daus le développement de la colonie. Minis. Agric. (luébec Circe, n 138.

Warakomska Z.. Igt)2. An investigation in to Pollen collections by Apis mellifica L. from two Different Parts of Poland. Ann. Univ. Mariae Curie Sklodowska, Sect. E, I'ologne, 17 (5), 67-1o6. 